\title{
Severe ceftazidime-induced drug reaction with eosinophilia and systemic symptoms (DRESS)
}

\author{
Matthieu Picard ${ }^{1 *}$, Philippe Bégin ${ }^{1}$, Jean Paradis ${ }^{1}$, Anne Des Roches $^{2}$, Louis Paradis ${ }^{1}$, Françoise Le Deist ${ }^{3}$ \\ From Canadian Society of Allergy and Clinical Immunology Annual Scientific Meeting 2011 \\ Quebec, Canada. 20-23 October 2011
}

\section{Background}

Drug reaction with eosinophilia and systemic symptoms (DRESS) is among the most severe forms of drug hypersensitivity [1]. Antiepileptics are by far the most commonly reported causative drugs [2]. Antibiotics have seldom been reported apart from minocycline [3].

\section{Case}

We describe a 55 years old female who developed DRESS with acute liver and kidney failure after being treated with ceftazidime and vancomycine. She was successfully treated with corticosteroids although while tapering prednisone she experienced a recurrence of the skin eruption without any systemic symptoms. She was taken off corticosteroids after 9 months of treatment.

\section{In vitro tests}

Fourteen months after the drug reaction, in vitro tests to identify the causal agent were performed. The lymphocyte transformation test (LTT) showed a marked proliferation to ceftazidime (stimulation index (SI): 17 at $100 \mathrm{mcg} / \mathrm{mL}$ ). CD25 was upregulated on CD4+ (induced expression: 17\%) and CD8+ (induced expression: 8\%) T cells as shown by flow cytometry when cultured with ceftazidime $50 \mathrm{mcg} / \mathrm{mL}$. IFN- $\gamma$ was markedly elevated in the supernatant of peripheral blood mononuclear cells (PBMC) cultured with ceftazidime $50 \mathrm{mcg} / \mathrm{mL}$ when compared to the control media (946 vs $13 \mathrm{pg} / \mathrm{mL}$ ). Vancomycin did not induce a significant response when compared to the control media in the flow cytometry and the IFN- $\gamma$ assays.

\section{Conclusions}

This is the first report of ceftazidime-induced DRESS to be subsequently proven by allergy tests. This case illustrates the importance of considering every susceptible drug as the potential etiologic agent. We also show the usefulness of in vitro tests in their identification.

\section{Author details \\ 'Department of medicine, Centre Hospitalier de I'Université de Montréal (CHUM), Montreal, Canada, H2L 4M1. 2Department of pediatrics, CHU Sainte- Justine, Université de Montréal, Montreal, Canada, H3T 1C5. ${ }^{3}$ Department of microbiology and immunology, CHU Sainte-Justine, Université de Montréal, Montreal, Canada, H3T 1C5.}

Published: 14 November 2011

References

1. Walsh SA, Creamer D: Drug reaction with eosinophilia and systemic symptoms (DRESS): a clinical update and review of current thinking. Clin Exp Dermatol 2011, 36:6-11.

2. Pirmohamed M, Friedmann PS, Molokhia M, et al: Phenotype standardization for immune-mediated drug-induced skin injury. Clin Pharmacol Ther 2011, 89:896-901.

3. Kano Y, Shiohara T: The variable clinical picture of drug-induced hypersensitivity syndrome/drug rash with eosinophilia and systemic symptoms in relation to the eliciting drug. Immunol Allergy Clin North Am 2009, 29:481-501.

doi:10.1186/1710-1492-7-S2-A37

Cite this article as: Picard et al:: Severe ceftazidime-induced drug reaction with eosinophilia and systemic symptoms (DRESS). Allergy, Asthma \& Clinical Immunology 2011 7(Suppl 2):A37. 